\title{
Flight Demonstration of Unmanned Aircraft System (UAS) Traffic Management (UTM) at Technical Capability Level 3
}

\author{
Arwa Aweiss \\ Aviation Systems Division \\ NASA Ames Research Center \\ Moffett Field, USA \\ Arwa.s.aweiss@nasa.gov
}

\author{
Jeffrey Homola \\ Human Machines Systems \\ NASA Ames Research Center \\ Moffett Field, USA \\ Jeffrey.r.homola@nasa.gov
}

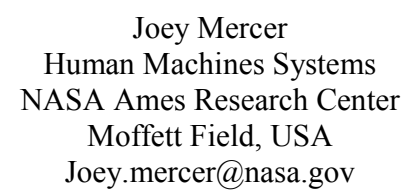

\author{
Joseph Rios \\ Aviation Systems Division \\ NASA Ames Research Center \\ Moffett Field, USA \\ Joseph.1.rios@nasa.gov
}

Hemil Modi

Science and Technology Corp

NASA Ames Research Center

Moffett Field, USA

hemil.c.modi-1@nasa.gov

\author{
Jaewoo Jung \\ Aviation Systems Division \\ NASA Ames Research Center \\ Moffett Field, USA \\ Jaewoo.jung@nasa.gov
}

\author{
Edgar Torres \\ Metis Flight Research \\ Associates, LLC \\ NASA Ames Research Center \\ Moffett Field, USA \\ edgar.torres@nasa.gov
}

\author{
Abraham Ishihara \\ SGT, Inc. \\ NASA Ames Research Center \\ Moffett Field, USA \\ abraham.k.ishihara@nasa.gov
}

\begin{abstract}
The goal of the Unmanned Aircraft System (UAS) Traffic Management (UTM) effort at NASA is to enable access to low-altitude airspace for small UAS. This goal is being achieved partly through partnerships that NASA has developed with the FAA, other government agencies, the UAS stakeholder community, and the designated FAA UAS Test Sites. This paper reports the technical and operational capabilities demonstrated during the UTM flight demonstration, March 6 through May 30, 2018. The demonstration featured geographically diverse operations, involving FAA UAS Test Sites in Alaska, Nevada, New York, North Dakota, Texas and Virginia. The demonstration leveraged the contributions of 30 partner organizations serving as UAS service suppliers, UAS operators, and/or providers of sensors, surveillance, connectivity, and management roles. Utilizing the UTM architecture developed at NASA, the demonstration explored 11 use cases for small UAS operations to highlight UTM capabilities at what NASA calls “Technical Capability Level (TCL) 3." TCL 3 is characterized by multiple small UAS safely operating in moderately populated areas and beyond the visual line of sight of their operators. The TCL 3 flights demonstrated the basic feasibility of such operations in the UTM environment, including USS exchanges; communication, navigation and surveillance functions; sense and avoid capabilities; and technologies and procedures to enable them.
\end{abstract}

\section{INTRODUCTION}

It is predicted that by 2022 there will be about 2.4 million hobbyists and over 450,000 commercial small Unmanned Aircraft Systems (sUAS) flying in the U.S. airspace [1]. These will be conducting diverse commercial, public safety, and hobbyist operations often beyond the visual line of sight of the operators. The vehicles may be in close proximity to each other, in different environments ranging from rural areas with sparse population to urban areas with dense population. No established infrastructure exists today to safely manage these operations. NASA's Unmanned Aircraft System (UAS) Traffic Management (UTM) project aims to enable access to low-altitude airspace for small UAS in a safe, efficient, scalable, and fair manner. NASA is collaborating with the FAA, UAS Test Sites, and industry to develop and test a UTM system research prototype to help identify requirements for an operational system. NASA has conducted its own flight demonstrations [2] and National Campaigns to assess the feasibility of operations at Technical Capability Levels (TCL) 1 and 2 [3-6]. The UTM Project partnered with six UAS Test Sites to perform UAS operations as part of the UTM "TCL 3 Flight Demonstration." TCL 3 is the third of four increasingly complex TCLs that support the entire range of sUAS operations. The TCL 3 environment involves enabling multiple beyond-visual-line-of-sight (BVLOS) operations, with some manned aircraft in proximity, and in suburban-like moderately populated areas. An overview of the flight demonstration, including tests sites, partners, and technical objectives, is presented in this paper. 


\section{BACKGROUND}

The expected increase in sUAS operations and proposed applications from industry and public safety entities was an impetus to the research and development of a UTM system [7-9]. In 2015, NASA and the FAA formed the UTM Research Transition Team (RTT) with the goal of identifying UTM capabilities and technologies that NASA will transfer to the FAA. There are four working groups: Concepts and Use Cases, Data Exchange and Architecture, Sense and Avoid, and Communication and Navigation. NASA presented and published an initial Concept of Operations in 2016 [10] and jointly with the FAA released an official UTM RTT Plan [11] and an updated Concept of Operations [12]. In addition to flight demonstrations, NASA has tested key components of the UTM system in simulation, especially data exchange between the components $[13,14]$.

UTM has become relevant outside of the United States. In 2015, European aviation stakeholders developed a Riga Declaration in which they stressed the need for the development of drone services, technologies, and standards to enable safe integration of drones into the European airspace [15]. A study sponsored by the Single European Sky ATM Research (SESAR) Joint Undertaking (JU) [16] led to the European Commission proposing to create an automated traffic management system for drones operating at low altitudes (referred to as the U-Space). In November 2017, the commission announced a push to speed up implementation of European Union drone rules [17]. SESAR JU forecasts indicate that all of the services planned as part of the European Union's UAS traffic management (UTM) program will be available by 2035, and that it will cost EUR 4.095 billion to create and will deliver around EUR 123 billion in benefits [17]. In March 2018, SESAR published its UTM Master Plan [18]. A paper was published describing the European approach to regulation and standardization and coordination with international and national agencies [19]. Then, in October 2018, the European Commission released a draft document for the regulation of the operation of unmanned aircraft [20]. The Concept of Operations for European UTM Systems (CORUS) project within the context of SESAR involves nine partner organizations and is tasked to detail the use of USpace to allow for socially acceptable and safe use of drones. The deliverable is scheduled for release in June 2019 [21].
Many industry partners have been involved in NASA's UTM efforts through participation in field demonstrations and NASA-FAA working groups [4, 10, 14]. Internationally, Unmanned Aircraft Systems Traffic Management (UTM) stakeholders have formed the Global UTM Association (GUTMA) consortium, which includes members from 20 countries [https://gutma.org/]. Eurocontrol has a list, "UTM Current State of the Art," of other UTM systems being developed worldwide [22]. The Japan Aerospace Exploration Agency (JAXA) collaborated with NASA in integrating their Integrated Aircraft Operation System for Disaster Relief (D-NET) with NASA's current UTM prototype as part of a research project. This was used to evaluate how the integrated system could efficiently be used to help maintain safety during relief operations [23].

The UTM Project is interested in accelerating stakeholder development of UTM components and in demonstrating scenarios across a wide range of operating environments, using the current UTM architecture, and with a wide range of UAS platforms and UAS Service Suppliers. In the UTM system, the UAS Service Supplier (USS) is a key component. Its main role is to support missions by the UAS operators. Its functions support the planning of UAS operations, provide information to UAS Operators during their operations, assist in strategic deconfliction of the UTM airspace, and bridge communication between UAS Operators and the Flight Information Management System (FIMS). The FIMS is a central, cloud-based component that acts both as a bridge to the existing National Airspace System (NAS) and as a broker of information between stakeholders. Connections to the FIMS are allowed from USSs that meet minimum requirements related to functionality, quality of service, and reliability [12].

\section{APPROACH}

Six FAA UAS Test Sites were contracted to develop scenarios for demonstration, evaluation, and refinement of concepts, technologies, and capabilities related to four main areas of research identified by the NASA/FAA Research Transition Team: Data Exchange and Information (DAT), Concept Use Cases (CON), Communication, Navigation, and Surveillance (CNS); Sense and Avoid (SAA). A total of 20 specific flight tests were designed to probe these research areas. 
The objective of the DAT focus area was to identify gaps in the UTM prototype model, refine Application Programming Interface (API) definitions, and define procedures during times of uncertainty such as a loss of a USS. Latencies such as those between USS submissions and exchange of information between the FIMS and USSs were investigated in the DAT scenarios.

The objective of the CON focus area was to refine the UTM concept for TCL 3 and its envisioned environment while also providing insight into the considerations necessary looking ahead to TCL4. Some examples of CON areas of interest included BVLOS landing accuracy and message timing for alerts for USS and sUAS such as when a sUAS is approaching a restricted airspace. Another was determining what UTM information should be publicly accessible. For this, some companies developed public portal applications.

The objective of the CNS focus was to explore ways to ensure that sUAS are under control of an operator and remain within a defined, authorized area. The CNS focus included communication and navigation system performance and radio frequency (RF) environment characterization. CNS technologies of interest included augmented navigation, direct and distributed communication (C2) links, and fourth generation long-term evolution (4G LTE) telecommunication.

The objective of the SAA focus area was to assess different separation mitigation strategies and interoperability of technologies. These included vehicle-to-vehicle (V2V), dedicated short-range communication (DSRC), and interaction between ground-based and airborne radar. Key research questions include (a) when should the timelines between USS mitigations and onboard mitigations overlap, and (b) what procedures should be in place to ensure separation in off-nominal conditions?

\section{METHOD}

The Flight Demonstration activities occurred between March 6 and May 30, 2018, encompassing 536 UAS sorties. Six FAA UAS Test Sites hosted the flight demonstration activities: Alaska, Nevada, New York, North Dakota, Texas and Virginia. Table 1 shows the distribution of the 536 completed flights at each test site. The sites conducted different sets of flight operations that probed some or all of the four focus areas. Each of the six test sites offered unique environments in which to demonstrate capabilities. For example, one site was at high altitude with ground obstacles of varying height. Another site included over 300 structures, open spaces, and lakes which helped emulate both suburban and rural areas. A variety of equipment and infrastructure at the sites and on the sUAS such as radars, LIDARs, and ADS-B were used to support the flight demonstration.

The sites partnered with 30 unique entities, including 13 sUAS operating companies, eight USS, five management and support companies, and eight companies providing sensor/surveillance/connectivity capabilities. Some of the companies participated as both operators and USSs. A total of 31 vehicles were flown (16 unique types) with a mix of multicopters, fixed wing, hybrids, and helicopters.

Different data collection methods were employed. The Data Management Plan (DMP) refers to the data collected by the UAS Test Sites, including ground control station, vehicle/operator, and surveillance data. The DMP data were uploaded to a NASA UTM website after the completion of the Flight Demonstration. The USSs submitted real-time data to the USS Data Collector (UDC).

Table 1. Number of Flights per Test Site

\begin{tabular}{|l|l|}
\hline Test Site & Number of Flights \\
\hline Alaska & 171 \\
\hline North Dakota & 69 \\
\hline Nevada & 60 \\
\hline New York & 60 \\
\hline Texas & 32 \\
\hline Virginia & 144 \\
\hline Total & 536 \\
\hline
\end{tabular}

\section{RESEARCH Focus AREAs DEMONSTRATED}

The objective of the UTM TCL 3 Flight Demonstration was to characterize the behavior of the UTM system components in terms of four key focus areas: Data Exchange and Information (DAT), Concept Use Cases (CON), Communication, Navigation, and Surveillnace (CNS), and Sense and Avoid (SAA)-Although not formally part of the TCL 3 Flight Demonstration, NASA also informally evaluated enabling 
technologies for sUAS operations including airborne and ground system functions [24] as they relate to the SAA and CNS focus areas.

\section{A. Data Exchange and Information (DAT)}

The goal of the six DAT tests was to exercise the system architecture-including the USSs, FIMS, and operators - to demonstrate that it could support identified concept use cases and had means to accommodate certain failures. The DAT are the following:

DAT 1: End-to-end UAS Reports (UREP)Operators submit UREPs (possibly through USS) during flights.

DAT 2: FIMS Failure- Simulate FIMS or a key FIMS component failing while there are active flights.

DAT 3: USS Failure- Simulate USS or a key USS component failing while there are active flights under its management.

DAT 4: Remote ID- Test UAS ID concept. Drone is registered with UTM Public Key Infrastructure (PKI) and broadcasts identity information. Received on ground and properly identified by authorities. DAT 5: USS-USS Negotiations- Operator A uses USS Y to plan and execute a mission. Operator B uses USS Z to plan and execute a mission. These missions overlap in some way. USS Y and USS Z use data exchanges to negotiate a resolution to the conflict in a strategic time horizon.

DAT 6: Weather Services- Use a UTM-focused weather service to aid in the planning of an operation. The weather data should be provided via a defined and published interface.

A UREP has a similar functionality to a Position Report (AIREP) or a Pilot Weather Report (PIREP) in today's conventional NAS operations. UREPs are data that are provided to the UTM System that can be shared with other stakeholders to provide better situational awareness. A UREP allows for reporting observable airspace activity as well as weather phenomena. The submission and retrieval of UREPs relies on automated processes [13].

Four test sites included the exchange of UREP messages in their demonstration activities. NASA and its partners at those sites used the UREP mechanism to exchange weather and airspace data. Over 26,000 UREPs were communicated via automated or manual processes to enhance operator awareness of airspace activity. Results to be presented in a future publication.

The Remote ID testing provided the first coordinated demonstration of a variety of technologies for identifying sUAS [25].

\section{B. Concept Use Cases (CON)}

The CON tests successfully demonstrated the following:

CON 1: Beyond-Visual-Line-of-Sight (BVLOS)

Landing

CON 2: Contingency Management

CON 3: Public Portal

CON 4: Multiple TCL 2/TCL 3 Operations for a Sustained Period

CON 5: FIMS/USS Interaction When a Vehicle Heads Towards Unauthorized Airspace.

CON 1 demonstrated the procedures and performance associated with conducting landings and subsequent takeoffs while operating BVLOS. Package deliveries while BVLOS were also performed as part of this test. CON 2 explored the response process and demonstrated the flow of information during contingency management responses to simulated off-nominal, emergency situations such as a loss of communication and navigation and how this would be communicated through the UTM system and the procedures involved. CON 3 showcased the concept of a "Public Portal": a prototype interface to access and visualize UTM data to improve situation awareness of operations. CON 4 required a scenario running for a few hours with multiple interacting TCL-2/3 operations (e.g. multiple deliveries, traffic monitoring, infrastructure inspection, real estate photography) so as to provide a window into complex operations in a dense airspace environment.

The CON 5 test addressed the concept area of interactions within the UTM architecture when a vehicle approaches and breaches specified areas of airspace, which were considered unauthorized airspace for the purposes of the demonstration. With respect to CON 5, three test sites performed operations as part of the test. Airspace structures utilized as part of the tests varied to include established Class E boundaries, static volumes instantiated prior to operations, and dynamic airspace volumes activated while UTM operations were already in progress. Regardless of the type of airspace structure used, the USS functionality 
demonstrated a phased alerting scheme where an operator first received a proximity warning message when the vehicle was within $3038 \mathrm{ft}(\sim 0.5$ nautical miles) of the airspace boundary. Another message was sent to the operator if and when the vehicle breached the bounds of the unauthorized airspace. Standard conformance monitoring protocols were also in place that first alerted the operator to Nonconforming or Rogue UTM states when vehicles were intentionally diverted to exit the Operation Volume and subsequently approach and enter the identified airspace as part of the testing protocol. Results are discussed in [28].

\section{Communication, Navigation, and Surveillance (CNS)}

During the TCL 3 Flight Demonstration, three aspects of CNS were demonstrated. In CNS 1, participating FAA UAS Test Site operators were directed to equip sUAS with redundant Command and Control (C2) systems, send a maneuver command via the primary system, and then verify execution of the sent command. This was repeated using the redundant system to demonstrate redundant command and control in maintaining operational control of sUAS. In CNS 2, operators were directed to fly sUAS into an area obstructed from GPS line of sight while tracking the sUAS's position with an independent ground truth system. This demonstrated the ability of sUASs to stay within their approved airspace even with the loss of onboard GPS signals. In CNS 3 , the operators were directed to fly sUAS with Radio Frequency (RF) channel-sensing payload. This was done to characterize the wireless RF communications environment at low altitude, focusing on LTE 4G frequency bands. In [26, 27], the design of the RF channel-sensing payload and the associated Electromagnetic Interference (EMI) test are discussed. Jung elaborates on the results of these CNS tests [29].

\section{Sense and Avoid (SAA)}

The sense and avoid testing consisted of six individual tests that demonstrated core challenges with keeping vehicles safely separated from other air traffic. To address the hazard associated with two sUAS having a mid-air collision, SAA 1 demonstrated air-to-air collision avoidance between two participating equipped sUASs using dedicated short-range communication (DSRC) radios to support vehicle-to-vehicle (V2V) communication. DSRC was more effective at close proximity $(\sim .5$ $\mathrm{km}$ or less) and under slow closure rates. Further optimization of radios, guidance of antennae position, and evaluation of required power output is expected to improve performance at faster speeds and farther communication ranges.

To address the hazard posed by transponderequipped (e.g. cooperative) manned aircraft, SAA 2 demonstrated successful resolution of lateral air-toair conflicts between a sUAS and manned aircraft using ADS-B "in" on the sUAS and ADS-B "out" on the manned aircraft. Resolution of vertical separation conflicts was not demonstrated using ADS-B.

SAA 3 and SAA 4 illustrated the challenge of using airborne or ground-based radar, respectively, to mitigate conflicts between sUAS and nontransponder-equipped (i.e., non-cooperative) manned aircraft. Low size, weight and power (SWaP) airborne radar did not provide sufficient range to support the recommended well-clear definition, had high false alerts, and may be better suited for collision avoidance rather than sense and avoid. Low SWaP ground-based radar demonstrated better performance and fewer false alerts than airborne radar, but multiple sensors may be needed to provide sufficient coverage.

These limitations highlight the rationale for the UTM's layered approach to conflict management. Airborne and ground-based separation mitigations are used in redundant layers spanning different time horizons. SAA 1-4 demonstrated different individual mitigation technologies or strategies to address key airborne hazards, while SAA 5 demonstrated their interoperability when combining different separation mitigation technologies on the same platform. The SAA 6 tests investigated whether DSRC could be extended to support air-to-ground UAS remote identification and obstacle avoidance.

\section{SUMMARY}

The UTM project at NASA has continued developing and testing a UTM system that will enable access to low-altitude airspace for small UAS. The TCL 3 Flight Demonstration was the third of four flight tests in the planned progression. A general overview of the demonstration and its outcomes was presented in this paper. The goal of TCL 3 was to demonstrate, evaluate, and refine the functional design and procedures, technology prototypes, and the UTM Concept of Operations.

The Flight Demonstration activities were focused on the four areas; Data Exchange and 
Information (DAT); Concept Use Cases (CON); Communication, navigation, and surveillance (CNS); and Sense and Avoid (SAA) which are priority areas of interest to the NASA-FAA Research Transition Team. These demonstrations were conducted during 33 flight days at six FAA UAS designated test sites: Alaska, Nevada, New York, North Dakota, Texas and Virginia. This provided a wide range of operating environments, USSs and UAS platforms. Eleven use cases were demonstrated, including package deliveries, infrastructure inspection, disaster response, coastal video surveys, and contingency management. Thirty unique entities partnered with the test sites to participate in the flight demonstrations. The TCL 3 Flight Demonstration successfully demonstrated the UTM technologies and procedures of safe sUAS operations given the assumptions and conditions of TCL 3. Lessons learned from TCL 3 contributed directly to the next flight demonstration, TCL 4, which are expected to take place in the summer of 2019.

\section{ACKNOWLEDGMENT}

The authors would like to thank the NASA UTM team members for their contribution to the TCL 3 Flight Demonstration. Thanks are also extended to the many dedicated individuals at the FAA UAS Test Sites and the organizations who partnered with them.

\section{REFERENCES}

[1] "FAA Releases Aerospace Forecast." [Online], Accessed: January 11, 2019. https://www.faa.gov/news/updates/?newsId $=89870$

[2] M. Johnson, J. Jung, J. Rios, J. Mercer, J. Homola, T. Prevot, D. Mulfinger, and P. Kopardekar, "Flight Test Evaluation of an Unmanned Aircraft System Traffic Management (UTM) Concept for Multiple Beyond-Visual-Line-of-Sight Operations," 12th USA/Europe Air Traffic Management Research and Development Seminar (ATM2017), Seattle, WA, June 26-30, $2017 .$.

[3] J. Rios, "NASA UAS Traffic Management National Campaign: Operations Across Six UAS Test Sites," 35th Digital Avionics Systems Conference (DASC), Sacramento, CA, 2016.

[4] A. S. Aweiss, B. Owens, J. L. Rios, J. Homola, and C. P. Mohlenbrink, "Unmanned Aircraft Systems (UAS) Traffic Management (UTM) National Campaign II," AIAA SciTech Forum, , Kissimmee, FL., January 8-12, 2018

[5] J. Homola, C. Mohlenbrink, Q. Dao, L. Claudatos, L. Martin, and Joey Mercer, "UAS Technical Capability Level 2 Unmanned Aircraft System Traffic Management (UTM) Flight Demonstration: Description and Analysis," IEEE-DASC, St. Petersburg, FL. September 17-21, 2017

[6] J. Jung, C. R. Drew, S. Nag, E. O. Torres, A. K. Ishihara, M. Do, and H. C. Modi, "Initial Approach to Collect Small Unmanned Aircraft System Off-nominal Operational Situations Data," AIAA Aviation 2018, Atlanta, GA., June 25-29, 2018.

[7] "Google UAS Airspace System Overview," presented at the 2015 UTM Convention at NASA Ames, Moffett Field, CA., [Online], Accessed:

January

11

2019.
https://utm.arc.nasa.gov/docs/GoogleUASAirspaceSystemOverview5 pager[1].pdf.

[8] "Determining Safe Access with a Best-Equipped, Best-Served Model for Small Unmanned Aircraft Systems," Amazon white paper, October, 2015, [Online], Accessed: January 12, 2019. https://utm.arc.nasa.gov/docs/Amazon_Determining\%20Safe\% 20Access $\% 20$ with $\% 20 \mathrm{a} \% 20$ Best-Equipped, $\% 20$ Best-Served $\% 20 \mathrm{Mod}$ el $\% 20$ for $\% 20$ sUAS[2].pdf.

[9] "Revising the Airspace Model for the Safe Integration of Small Unmanned Aircraft Systems," Amazon white paper, October, 2015, [Online], Accessed: January 12, 2019]. https://utm.arc.nasa.gov/docs/Amazon_Revising\%20the\%20Airspace \%20Model $\% 20$ for $\% 20$ the $\% 20$ Safe $\% 20$ Integration $\% 20$ of $\% 20$ sUAS 6 ].pdf.

[10] P. Kopardekar, J. Rios, T. Prevot, M. Johnson, J. Jung, and J. E. Robinson III, "Unmanned Aircraft System Traffic Management (UTM) Concept of Operations," presented at the 16th AIAA Aviation Technology, Integration, and Operations Conference, Washington, DC. , June 13-17, 2016.

[11] "UAS Traffic Management (UTM) Research Transition Team (RTT) Plan 2017", [Online], Accessed: January 12

2019. https://www.faa.gov/uas/research development/traffic manage ment/media/FAA_NASA_UAS_Traffic Management Research Plan .pdf

[12] "Unmanned Aircraft System Traffic Management (UTM) Concept of Operations," Federal Aviation Administration, Washington, DC, May 18, 2018 [Online], Accessed: January 15, 2019 https://utm.arc.nasa.gov/docs/2018-UTM-ConOps-v1.0.pdf

[13] J. L. Rios, I. S. Smith, and D. R. Smith, "NASA Technical Memorandum-UAS Reports (UREPs): Enabling Exchange of Observation Data Between UAS Operations," Moffett Field, CA, February, 2017.

[14] J. L. Rios, D. G. Mulfinger, I. S. Smith, P. Venkatesan, D. R. Smith, V. Baskaran, and L. Wang, "NASA Technical Memorandum-UTM Data Working Group Demonstration 1 Final Report," Moffett Field, CA, April, 2017.

[15] Riga Declaration on Remotely Piloted Aircraft Systems, "Framing the future of aviation," Riga, Latvia, March 6, 2015, [Online], Accessed: $\quad$ January 2019]. https://ec.europa.eu/transport/sites/transport/files/modes/air/ne ws/doc/2015-03-06-drones/2015-03-06-riga-declaration-drones.pdf

[16] SESAR (Single European Sky ATM Research) Joint Undertaking, "Europeans Drones Outlook Study: Unlocking the value for Europe," November, 2016, [Online], Accessed: January 12, 2019. https://www.sesarju.eu/sites/default/files/documents/reports/European Drones Outlook Study 2016.pdf

[17] Florian Guillermet, "High-level plan to integrate all categories of drones in Europe," presented at the High level Conference on Drones, Helsinki, November 2017, [Online], Accessed: January 12, 2019. https://www.sesarju.eu/sites/default/files/documents/events/HighLeve lConferenceDrone-FG.pdf

[18] Florian Guillermet, "European UTM Master Plan: Roadmap for the safe integration of drones into all classes of airspace," March 8, 2018, [Online], Accessed: January 10, 2019. https://www.sesarju.eu/sites/default/files/documents/reports/European \%20ATM\%20Master\%20Plan\%20Drone\%20roadmap.pdf

[19] ICAO Working Paper, "UTM Integration in Europe: Roadmap for the safe integration of drones into all classes of airspace," presented at the Thirteenth Air Navigation Conference, Montreal, Canada, October 9-19, 2018, [Online], Accessed: January 10, 2019. https://www.icao.int/Meetings/anconf13/Documents/WP/wp_051_en. pdf

[20] Commission Implementing Regulation on rules and procedures for the operation of unmanned aircraft (draft) and Annex October 8, 2018, [Online], Accessed: January 10, 2019. https://ec.europa.eu/info/law/betterregulation/initiatives/2018drones_en

[21] "Concept of Operations for EuRopean UTM Systems", [Online], Accessed: January 2019. https://trimis.ec.europa.eu/project/concept-operations-european-utmsystems\#tab-outline

[22] http://www.eurocontrol.int/articles/utm-current-state-art

[23] J. Homola, M. Johnson, P. Kopardekar, A. Andreeva-Mori, D. Kubo, K. Kobayashi, and Y. Okuno, "UTM and D-NET: NASA and JAXA's 
Collaborative Research on Integrating Small UAS with Disaster Response Efforts," 2018 Aviation Technology, Integration, and Operations Conference, AIAA AVIATION Forum, Atlanta, GA., June 25-29, 2018.

[24] R. Kerczewski, R. D. Apaza, A. N. Downey, J. Wang, and K. J. Matheou, "Assessing C2 Communications for UAS Traffic Management," ICNS 20, Herndon, VA, April 10-12, 2018.

[25] A. Ishihara, J. Rios, and P. Venkatesan, "Remote ID for Rapid Assessment of Flight and Vehicle Information," AIAA 2019 SciTech Forum, San Diego, CA, January 7-11, 2019.

[26] J. Jung, C. Ippolito, C. Rogers, R. Kerczewski, Downey, and K. Matheou, "Small Unmanned Aircraft Electromagnetic Interference (EMI) Initial Assessment," ICNS 2018, Herndon, VA, April 10-12, 2018.

[27] A. J. Moore, S. Balachandran, S. Young, E. Dill, M. J. Logan, L. Glaab, C. Munoz, and M. Consiglio, "Test for Enabling Technologies for Safe
Urban Operations," presented at the 2018 AIAA Aviation Technology, Integration, and Operations Conference, Atlanta, GA, June 25-29, 2018.

[28] J. Homola, L. Martin, M. Cencetti, and A. Aweiss, "UAS Traffic Management (UTM) Technical Capability Level 3 (TCL3) Flight Demonstration: Concep Tests and Results," $38^{\text {th }}$ DASC 2019, San Diego, CA, September 8-12, 2019. , in press.

[29] J. Jung, S. Nag, H. C. Modi, "Effectiveness of Redundant Communications Systems in Maintaining Operational Control of Small Unmanned Aircraft," $38^{\text {th }}$ DASC 2019, San Diego, CA, September 812,2019 . , in press. 